\title{
INQUÉRITOS DE SAÚDE E FONOAUDIOLOGIA
}

\section{Health surveys and audiology and speech-language science}

\author{
Mariana Sodário Cruz ${ }^{(1)}$, Luiz Roberto de Oliveira ${ }^{(2)}$, Luana Carandina ${ }^{(3)}$
}

\begin{abstract}
RESUMO
Tema: os inquéritos de saúde constituem uma abordagem eficiente para estudos populacionais, sendo tal metodologia ainda pouco difundida entre os fonoaudiólogos brasileiros. Para a realização e análise de um estudo com base em inquéritos de saúde, o profissional deve transcender a visão clínica fonoaudiológica, partindo para uma abordagem social, demográfica, econômica e de saúde geral. Objetivo: realizar abordagem teórica acerca dos inquéritos de saúde, seus conceitos, histórico e resultados publicados no campo fonoaudiológico, com enfoque em saúde auditiva. Conclusão: os inquéritos de saúde constituem-se num método de investigação importante a ser explorado pelos fonoaudiólogos para que passem a recomendar ações relacionadas à saúde auditiva em sintonia com necessidades da população embasadas em dados epidemiológicos fidedignos.
\end{abstract}

DESCRITORES: Inquéritos de Morbidade; Perda Auditiva; Saúde Pública

\section{INTRODUÇÃO}

A atuação fonoaudiológica em saúde coletiva pode ser considerada como um campo em grande expansão, com profissionais em busca de sua identidade e de conhecimentos específicos para atuação na área, tanto na prestação de serviços, como na gestão e administração de bens de saúde coletivos. Para tal finalidade, faz-se necessária a busca do saber em áreas distintas à sua, como, por exemplo, a Medicina, Geografia, Administração, Ciências Sociais, Ciências Políticas, História, entre outros. Essa busca é desejável para um trabalho completo, devendo ser, inclusive, estimulada. Entretanto, também pode indicar a escassa produção científica

(1) Fonoaudióloga da Prefeitura Municipal de Itatinga, SP; Mestre em Saúde Coletiva pela Universidade Estadual Paulista.

(2) Médico; Professor Assistente Doutor do Departamento de Saúde Pública da Faculdade de Medicina de Botucatu da Universidade Estadual Paulista, UNESP, Botucatu, SP; Doutor em Saúde Pública pela Universidade de São Paulo.

(3) Médica; Professora Assistente Doutora do Departamento de Saúde Pública da Faculdade de Medicina de Botucatu da Universidade Estadual Paulista, UNESP, Botucatu, SP; Doutora em Saúde Pública pela Universidade de São Paulo.

Trabalho realizado no Departamento de Saúde Pública da Faculdade de Medicina de Botucatu-SP/FMB-UNESP, Programa de Pós Graduação em Saúde Coletiva, como parte da dissertação de mestrado da primeira autora. Data da defesa: 07/05/2007. fonoaudiológica em Saúde Coletiva no Brasil, visto que poucas publicações da área abordam modelos conceituais ou discutem possibilidades de pesquisa com base em propostas epidemiológicas populacionais, na contramão do grande número de pesquisas clínicas realizadas, segundo levantamento realizado nas bases de dados Medline, Lilacs e Scielo, no período de 2000 a 2007.

A visão fonoaudiológica predominantemente curativa ainda persiste na atuação de alguns profissionais inseridos em serviços de saúde pública, preocupados em atender toda a demanda, geralmente espontânea, de pacientes que procuram as Unidades Básicas de Saúde, Hospitais, Centros de Referência, entre outros. Este tipo de abordagem geralmente caminha para a frustração profissional, visto que o acolhimento de toda a demanda pode se tornar algo inatingível em alguns serviços, que passam a formar longas filas de espera para o atendimento, transmitindo a idéia de que o trabalho fonoaudiológico é acessível para poucos.

Essa abordagem excludente remete ao histórico da Fonoaudiologia no Brasil, que se iniciou no ambiente escolar na década de 20 do século passado, quando da determinação do patológico e saudável em uma nação povoada de imigrantes, com inúmeras maneiras de se comunicar. Vale ressaltar que esta abordagem tinha foco nacionalista, ou seja, valiam as regras do Português nacional, fixando, no indivíduo, a "culpa" de suas patologias e ignorando o meio social em que este se inseria ${ }^{1}$. 
Nas décadas seguintes, a atuação fonoaudiológica seguiu para consultórios particulares e clínicas de reabilitação, ainda com o enfoque individualista da saúde comunicativa. A dificuldade em se atuar de maneira coletiva se devia à não inserção deste profissional em serviços públicos e, também, ao conceito de saúde vigente na época: "bem estar físico, social e mental, não somente a ausência de doenças". Esta conceituação dificultava as ações de saúde pública, visto que a saúde se configurava como um estado inalcançável ${ }^{2}$.

A partir da regulamentação da profissão, ocorrida com a aprovação da Lei 6965 de 09 de dezembro de 1981, foi estabelecido um currículo mínimo de formação, inicialmente considerado "tecnicista", com a preocupação em se formar profissionais capazes de tratar distúrbios já instalados. A partir da Resolução CNE/CES 05 de 19 de fevereiro de $2002{ }^{3}$, que instituiu as Diretrizes Curriculares Nacionais dos Cursos de Graduação em Fonoaudiologia, esta postura sofreu profundas transformações. Tal documento preconizou a necessidade do fonoaudiólogo ser capaz de trabalhar em atenção à saúde, tanto de forma individual quanto coletiva, com liderança, desenvolvendo atividades de cunho administrativo. A necessidade desta mudança na formação fonoaudiológica já vinha sendo percebida nos anos 90 , a partir da inserção do fonoaudiólogo nos serviços públicos, momento em que estes profissionais começaram a perceber a necessidade da mudança das concepções de saúde até então utilizadas como parâmetros.

Atualmente, há grande mobilização de profissionais da área para a divulgação e discussão dos temas relacionados à Saúde Pública e Coletiva, com ingresso de fonoaudiólogos em cursos de Pós-Graduação em Saúde Coletiva e até mesmo a inclusão do título de especialista em Saúde Coletiva como titulação reconhecida pelo Conselho Federal de Fonoaudiologia. Esta última atitude vem coroar a percepção de que, para um fonoaudiólogo atuar em Saúde Coletiva não basta que ele saiba trabalhar conjuntamente com todas as áreas fonoaudiológicas, mas também tenha conhecimentos suficientes em Saúde Coletiva e em suas subáreas incluindo a epidemiologia. Deste modo, o objetivo do presente estudo foi realizar revisão da literatura acerca dos inquéritos de saúde, seus conceitos, histórico e resultados publicados no campo fonoaudiológico, com enfoque em saúde auditiva.

Os inquéritos de saúde de base populacional são usados mais largamente em países desenvolvidos e ilustram a condição de vida e saúde de populações num determinado momento. É uma maneira considerada economicamente viável de se estudar grande número de pessoas e de se obter dados necessários à formulação de políticas públicas, podendo contribuir de forma ímpar para o planejamento em saúde dos serviços fonoaudiológicos no país.

Foi realizada pesquisa bibliográfica em artigos de periódicos nacionais e internacionais, livros e documentos eletrônicos considerando-se a relevância e o valor didático de tais materiais para o assunto em estudo. Foram consultados livros científicos, sites e periódicos da área de Fonoaudiologia, Otorrinolaringologia e Saúde Coletiva, além de busca nas bases de dados Medline, Lilacs e Scielo com os descritores "morbidity surveys", "hearing loss" e "social conditions", considerando os trabaIhos a partir de 1980.

O tema foi desenvolvido segundo tópicos conceituais que abrangeram: epidemiologia, inquéritos de saúde e sua relação com a Fonoaudiologia, por meio de uma abordagem histórica e didática sobre o tema; condições de vida e deficiência auditiva, que abordou como os conceitos de condições de vida podem se relacionar e auxiliar pesquisas sobre deficiência auditiva e, para finalizar, o panorama atual e perspectivas futuras de pesquisas fonoaudiológicas com base em inquéritos de saúde.

\section{REVISÃO DA LITERATURA}

\section{Epidemiologia descritiva, inquéritos de saúde e Fonoaudiologia}

A epidemiologia descritiva tem por objetivo ilustrar dados existentes em uma determinada população, com a finalidade de conhecê-la melhor sob algum determinado aspecto e, deste modo, nortear intervenções que abranjam necessidades reais desta população alvo. Entre as várias metodologias possíveis de serem empregadas, destaca-se a utilização de inquéritos de saúde, que possibilitam resultados consistentes e de grande relevância para o planejamento, formulação e avaliação de políticas e programas de saúde ${ }^{4}$.

Os inquéritos epidemiológicos são ferramentas para detectar a presença de agravos à saúde na população, informando sobre freqüência e distribuição, assim como sobre numerosos outros aspectos associados. Uma alternativa simples é realizá-lo por entrevistas (domiciliares, telefônicas, por correspondência), na tentativa de certificar-se sobre as afecções de que uma pessoa foi ou está acometida e, em alguns momentos, sobre óbitos na família que possam ser atribuídos a doenças infecciosas ${ }^{5}$, gerando taxas de morbidade e mortalidade. Estas têm sido consideradas indicadores confiáveis das condições de saúde populacionais e reveladoras das desigualdades entre grupos estudados ${ }^{6}$. 
Quando o inquérito ocorre sob a forma de um estudo transversal, ou seja, representando um único momento, pode ser considerado como uma "fotografia" da realidade, ou seja, apenas descreve situações instantâneas, não possibilitando o estabelecimento preciso das relações de causalidade das morbidades estudadas. Por outro lado, uma vantagem deste tipo de estudo é que os bancos de dados criados permitem uma análise mais abrangente dos serviços de saúde, ao incorporarem informações sobre populações atendidas e não atendidas, permitindo até mesmo a caracterização desta demanda segundo variáveis como sexo, idade, local de moradia e condições sócio-econômicas ${ }^{4}$.

Historicamente, o primeiro inquérito populacional reportado ocorreu em 1842, num estudo comparativo entre o estado de saúde de trabalhadores britânicos cobertos pelo seguro saúde e presidiários ${ }^{7}$. Posteriormente, no final dos anos 50 , estes começaram a ser realizados de forma periódica nos Estados Unidos da América, mesclando, em alguns casos, entrevistas com exames clínicos ${ }^{8}$.

No Brasil, os inquéritos nacionais começaram a ser aplicados a partir dos anos $70 \mathrm{com}$ o Estudo Nacional de Despesa Familiar (ENDEF), as Pesquisas de Orçamento Familiar (POF) e a Pesquisa Nacional sobre Saúde e Nutrição (PNSN), além da Pesquisa Nacional por Amostras de Domicílios (PNAD), a qual, devido à maior abrangência e periodicidade, possui maior importância ${ }^{8}$.

Nos anos 80 , ocorreram os primeiros inquéritos sobre morbidade referida e uso de serviços de saúde, de âmbito municipal, em localidades do interior do Estado de São Paulo, tais como Ribeirão Preto ${ }^{9}$ e Botucatu ${ }^{10}$. Posteriormente, nos anos de 2001 a 2003, foi realizado um inquérito que enfocou quatro áreas do Estado de São Paulo: região de Itapecerica da Serra na Grande São Paulo, Distrito do Butantã no município de São Paulo, municípios de Campinas e Botucatu, o inquérito de saúde ISA-SP. Esse estudo caracterizou as condições de vida e saúde das populações dessas áreas e abordou dados relativos à morbidade, renda, escolaridade, condições de moradia, acesso aos serviços de saúde, presença de deficiências físicas, entre outros ${ }^{11}$.

Metodologicamente, a realização de inquéritos populacionais exige, a priori, o estabelecimento de indicadores eficazes para o recorte da amostra a fim de que a realidade social seja apreendida de maneira representativa. Com este objetivo, renda média familiar, infra-estrutura sanitária de água e esgoto, número de filhos entre mulheres de 15 a 49 anos, proporção de pessoas idosas, escolaridade do chefe da família, idade e sexo dos entrevistados ${ }^{11,12}$, dentre outros aspectos, tem sido utilizados.
A partir da predominância dos indicadores eleitos para o estudo, são definidas diversas áreas tomadas como homogêneas e que comporão o universo a ser amostrado. Para a definição destas áreas homogêneas, muitos estudos utilizaram os dados e as divisões censitárias estabelecidas pelo IBGE Instituto Brasileiro de Geografia e Estatística e as amostras extraídas puderam contemplar as diferentes condições sociais da população, tais como os inquéritos realizados em Ribeirão Preto ${ }^{9}$, Botucatu ${ }^{10}$ e o estudo ISA-SP ${ }^{11}$.

No campo fonoaudiológico, a utilização de inquéritos de saúde pode ser encontrada, mais comumente, em estudos dirigidos à saúde auditiva. O uso de questionários para o levantamento de taxas de deficiência auditiva tem se mostrado um instrumento de grande utilidade, principalmente nos casos em que o uso de medidas quantitativas (por exemplo, audiometria) não é viável ${ }^{13}$.

Em países em desenvolvimento, a utilização de questionário pode ser uma opção financeiramente vantajosa para a detecção de perdas auditivas, principalmente em pré-escolares ${ }^{14}$. No entanto, é importante observar a idade do entrevistado, o conhecimento da população acerca de sua saúde auditiva, quais perguntas serão utilizadas e os termos empregados. Por exemplo, para a averiguação da saúde auditiva em crianças (considerando até 12 anos de idade), faz-se necessária a utilização de um instrumento específico, que aborde, além da questão direta sobre audição, dados sobre o desenvolvimento de fala e linguagem, comportamento infantil e presença de otites médias ${ }^{15}$.

No Brasil, estudos populacionais sobre audição ilustram mais freqüentemente, achados sobre os idosos ${ }^{16,17}$. Esse fato pode ser explicado devido à presbiacusia configurar-se como uma das causas mais freqüentes de deficiência auditiva adquirida, juntamente com as causas relacionadas ao trabaIho ${ }^{18}$. Outra população que recebe destaque nos estudos de prevalência de alterações auditivas são os escolares 19,20. Esses estudos são de extrema importância para o conhecimento da saúde auditiva de crianças e adolescentes, entretanto, por avaliarem somente as crianças inseridas no ambiente escolar não é possível, a partir desses achados, determinar ou extrapolar taxas de prevalências para a população como um todo, em igual faixa etária, considerando que a amostra é de uma determinada instituição ou, ainda, que podem estar distorcidos pela evasão escolar existente no Brasil, que atinge justamente crianças e adolescentes mais carentes.

Com a finalidade de determinar mais precisamente as taxas de prevalência de deficiência auditiva no país, grupos de pesquisa em audiologia começaram a desenvolver estudos populacionais 
de maior porte. Em 2003 foi realizado um trabalho pioneiro sobre audição no Brasil, com 2427 sujeitos acima de quatro anos de idade ${ }^{21}$, que consistiu da aplicação de um inquérito domiciliar e de avaliações audiométricas para a averiguação da saúde auditiva da população de Canoas-RS. Foi encontrada prevalência de $6,8 \%$ de deficiência auditiva, por meio do instrumento "WHO Ear and Hearing Disorders Survey Protocol" da Organização Mundial da Saúde. Esse mesmo instrumento também está sendo utilizado num estudo populacional realizado em Monte Negro-RO, que tem por objetivo determinar a prevalência de deficiência auditiva na população urbana desta localidade, através da avaliação de 1.345 pessoas de um universo de 5.722 habitantes distribuídos em setores censitários, trabalho este que se encontra em fase de publicação.

Estudos realizados em vários países para quantificar a sensibilidade e especificidade de determinadas questões e protocolos para o levantamento da deficiência auditiva em grandes populações, compararam a deficiência referida com a comprovada por meio de medidas audiométricas. Dentre esses, destaca-se "The Blue Mountains Hearing Study" ${ }^{22}$, realizado na Austrália. Segundo o referido estudo, tanto o questionário HHIE-S (Hearing Handicap Inventory for the Elderly: screnning version) quanto a questão "do you feel you have a hearing loss?' apresentaram sensibilidade e especificidade satisfatórias para a estimativa de deficiência auditiva na população entre 55 e 99 anos.

Além da escolha da questão ou do protocolo a ser utilizado para o correto levantamento epidemiológico, outro fator pode interferir nos resultados de estudos de morbidade referida: a auto-percepção da doença e/ou deficiência. A percepção de doença e de saúde está ligada ao desempenho dos papéis sociais das pessoas, ou seja, devido à menor exposição aos cuidados de saúde, as classes menos privilegiadas compreenderiam a doença de forma mais precária e, por isso, usariam vocabulário muito mais restrito e teriam percepção mais limitada da doença ${ }^{23}$. Enquanto as pessoas mais bem situadas socialmente seriam alertadas pelos primeiros sintomas de uma doença, nas classes menos favorecidas a doença tenderia a ser levada em consideração apenas quando interferisse no uso pleno do corpo, normalmente para o trabalho ${ }^{24}$.

Desse modo, estima-se que deficiência auditiva seja uma das condições crônicas de maior prevalência sem diagnóstico, ao lado da depressão e atrite. Suspeita-se que até $50 \%$ de indivíduos com relato de deficiência auditiva não sejam submetidos à avaliação profissional, achado mais significativo entre os idosos ${ }^{25}$ que esperariam até 10 anos entre o aparecimento da deficiência e a procura de ajuda profissional ${ }^{26}$. Uma explicação possível para este achado seria a dificuldade de acesso aos serviços de saúde especializados, o que acarretaria diagnósticos tardios. Estes achados indicam que taxas de prevalências de deficiências auditivas calculadas a partir de dados institucionais, ou seja, tendo por base somente os sujeitos que chegaram ao serviço de saúde específico, estão, provavelmente, subestimadas.

\section{Condições de vida e deficiência auditiva}

O levantamento das condições de vida por meio dos inquéritos de saúde tem se tornado um ponto importante nos estudos epidemiológicos devido, principalmente, à influência sócio-ambiental no processo saúde-doença. Segundo o Ministério da Saúde ${ }^{27}$ a saúde é um processo dinâmico, uma dimensão da qualidade de vida, um direito humano fundamental para o desenvolvimento de uma nação. Nesta visão, a saúde deixa de ser competência somente de profissionais e instituições específicas e passa a ser responsabilidade de diversos setores da sociedade envolvidos com a melhoria da qualidade de vida, como a educação, habitação, saneamento básico, cultura e abastecimento.

Entre os aspectos mais amplamente abordados para um estudo das condições de vida em populações alvo destacam-se escolaridade, rendimentos (familiares, do chefe de família ou per capita) e ocupação.

A escolaridade pode estabelecer associações com as taxas de prevalência de deficiência auditiva, principalmente na população adulta, visto que essas taxas tendem a aumentar conforme ocorre a diminuição do nível educacional ${ }^{18}$. Entretanto, no caso de se considerar a população idosa, a escolaridade perde a significância estatística como fator associado ao aumento da prevalência de deficiência auditiva, sendo que esta se eleva independente do nível educacional do sujeito em estudo ${ }^{28}$. Além da prevalência da deficiência auditiva aumentar em níveis escolares menos favorecidos, também se deve ressaltar a dificuldade de acesso à educação do sujeito com este tipo de deficiência. Segundo dados do MEC - Ministério da Educação e Cultura, dentre os 406 mil deficientes auditivos em idade escolar no Brasil, somente 56 mil (13\%) cursaram a educação básica no ano de 2003. No ensino médio este número caiu para dois mil e, nas universidades, para $300^{29}$.

Quanto aos rendimentos, pode-se averiguar que a prevalência da deficiência auditiva tende a cair na medida em que a renda familiar aumenta, dados relatados em estudos brasileiros ${ }^{21}$ e internacionais. Por exemplo: famílias com renda anual inferior a 20.000 
dólares/ano seriam duas vezes mais propícias a apresentar perda de audição em comparação àquelas que têm rendas superiores a $50.000{ }^{28}$.

A ocupação possui um lugar de destaque no estudo da deficiência auditiva, pois, para um deficiente auditivo, a inclusão no mercado de trabalho ainda é um desafio a ser superado e, por outro lado, o trabalho, ou as condições deste, pode ser o desencadeador da deficiência auditiva. Em um estudo realizado na Suécia ${ }^{30}$, concluiu-se que ações coordenadas, assim como políticas públicas associadas a medidas de segurança no trabalho, podem reduzir a discrepância entre ouvintes e deficientes auditivos quanto à escolha e desempenho profissional.

Tomando-se a ocupação como etiologia da deficiência auditiva, considera-se esta uma doença ocupacional de alta prevalência nos países industrializados, destacando-se como um dos agravos à saúde do trabalhador mais prevalentes. O mesmo observa-se nas indústrias brasileiras. A perda auditiva do tipo neurossensorial e irreversível ocorre, mais significantemente, após seis anos de exposição a elevados níveis de pressão sonora ${ }^{31}$.

Além da escolaridade, renda e ocupação, as condições de vida, de forma geral, em países subdesenvolvidos, também corroboram para o aumento das taxas de prevalência da deficiência auditiva. Esse fato se deve à maior ocorrência de doenças de orelha média, problemas congênitos, doenças infecciosas, além de identificação tardia de perdas leves e moderadas, mais comuns em países menos privilegiados economicamente ${ }^{32}$. Este achado ressalta a importância de estudos epidemiológicos locais, ou seja, brasileiros, para o estabelecimento de políticas públicas de saúde auditiva, que atendam às reais necessidades da população em todos os níveis de prevenção.

\section{Inquéritos de Saúde e Fonoaudiologia: panorama atual e perspectivas futuras}

O Brasil possui poucas publicações que abordam a saúde auditiva por meio de inquéritos e, em sua grande maioria, estes poucos estudos são fragmentos de grandes pesquisas de morbidade que avaliaram outros aspectos além da deficiência auditiva. Este fato se deve, primeiramente, ao custo da realização de um inquérito e a todo o trabalho envolvido na elaboração de pesquisas deste porte, sendo que, quando universidades ou centros de pesquisas os realizam, geralmente aproveitam para o estudo de diversas afecções. O ponto negativo deste tipo de situação é que, muitas vezes, são utilizadas questões simples para a averiguação da deficiência auditiva, questões ainda não padronizadas no Brasil, além do fato de que pontos importantes relativos a esta deficiência tendem a ser ignorados, tais como: histórico de saúde auditiva (infecções, doenças específicas), acesso ao aparelho de amplificação sonora individual e histórico do desenvolvimento da linguagem ${ }^{11,16,17}$.

Outro fator de extrema importância para a realização de estudos audiológicos populacionais seria a confirmação dos relatos de deficiência auditiva por meio de medidas audiométricas, método aplicado em apenas um trabalho publicado no Brasil ${ }^{21}$. Por mais que estudos confirmem a sensibilidade e especificidade de questões isoladas para o levantamento da deficiência auditiva ${ }^{13,14,22}$, quando não há a confirmação objetiva, por exemplo, audiométrica, deve-se aceitar que os valores encontrados somente se relacionam à deficiência auditiva referida, sendo esta uma limitação dos estudos dessa natureza.

Entretanto, as iniciativas destes estudos mais genéricos em abordar a deficiência auditiva devem ser estimuladas, pois possibilitam o cruzamento de dados entre a deficiência auditiva e outras afecções, além de abrirem o caminho para estudos aprofundados sobre saúde auditiva, visto que traçam um panorama geral e indicam a necessidade de estudos específicos para maior detalhamento destas questões.

Nesse campo de estudo, a Fonoaudiologia brasileira, em parceria com as demais profissões que estudam a audição (Otorrinolaringologia, Psicologia, Serviço Social, Enfermagem, entre outros) deve aprofundar a aplicação de inquéritos sobre saúde auditiva no nosso país a fim de conhecer melhor a realidade brasileira dos deficientes auditivos e propor políticas públicas de saúde condizentes com essas necessidades. Esses profissionais devem atuar tanto na elaboração dos inquéritos - desde a escolha das questões a serem abordadas, avaliando a sensibilidade e especificidade das mesmas como na aplicação dos inquéritos e análise dos resultados, discutindo pontos relativos a condições de vida desta população. Espera-se que o fonoaudiólogo, pela investigação das características e necessidades da população, tenha conhecimentos suficientes para realizar reflexões críticas sobre as políticas de saúde vigentes em nosso país, podendo contribuir para aprimorá-las.

\section{COMENTÁRIOS FINAIS}

Inquéritos epidemiológicos podem fornecer à Fonoaudiologia, assim como a todas as demais profissões da saúde, dados importantes para a atuação preventiva junto a determinadas populações. Em comparação aos países desenvolvidos, - Brasil ainda possui uma produção científica 
modesta no campo dos inquéritos populacionais, principalmente no que se refere ao estudo da prevalência de deficiência auditiva e dos fatores que podem se relacionar a esta deficiência, tais como condições de vida, saúde geral e acesso a serviços de saúde. Desse modo, este tipo de estudo se configura como um campo científico importante a ser explorado pelos profissionais da área para que as ações de saúde propostas pelos fonoaudiólogos contemplem a necessidade real da população em relação a sua saúde auditiva e sejam embasadas em dados epidemiológicos fidedignos.

\section{ABSTRACT}

Background: health surveys make up an efficient approach for population researches, and this methodology is not widely diffused among Brazilian audiologists and speech-language pathologists. In order to carry out a research based on health surveys; the professional should transcend the clinical audiologist and speech-language pathologist view, aiming at a social, demographic and economic view of health. Purpose: achieve theoretic approach about health inquiries, their concepts, history and published results in audiology and speech-language science, with emphasis on hearing health. Conclusion: health surveys make up an important investigation method to be explored by audiologists and speech-language pathologists, so that they mat recommend actions related to hearing health according with the population needs based on reliable epidemiologic data.

KEYWORDS: Morbidity Surveys; Hearing Loss; Public Health

\section{REFERÊNCIAS}

1. Berberian AP. Fonoaudiologia e educação: um encontro histórico. 1. ed. São Paulo: Plexus; 1995.

2. Penteado RZ, Servilha EAM. Fonoaudiologia em saúde pública/coletiva: compreendendo prevenção e o paradigma da promoção da saúde. Rev Dist Comun. 2004; 16(1):107-16.

3. Brasil. Resolução CNE/CES 5 2002. Disponível em: URL: http://www.crfono4r.com.br/fono.shtml. Acesso em 3 mar 2007.

4. Cesar CLG, Tanaka OY. Inquérito domiciliar como instrumento de avaliação de serviço de saúde: um estudo de caso da região sudoeste da área metropolitana de São Paulo, 1989-90. Cad Saúde Publica. 1996; 12(2):59-70.

5. Pereira MG. Doenças infecciosas. In: Pereira MG. Epidemiologia: teoria e prática. Rio de Janeiro: Guanabara Koogan; 1995. p. 429.

6. Gomes KRO, Tanaka AC. Morbidade referida e uso dos serviços de saúde por mulheres trabalhadoras, Município de São Paulo. Rev Saúde Pública. 2003; 37(1):75-82.

7. Campos CEA. Os inquéritos de saúde sob a perspectiva do planejamento. Cad Saúde Pública. 1993; 9(2):190-200.

8. Barros MBA. Introdução: os inquéritos domiciliares e o estado de saúde da população. In: Chester LG, César CLG et al. Saúde e condição de vida em São Paulo. São Paulo: USP; 2005. p. 11-34.
9. Carvalheiro JR. Investigação epidemiológica e entrevistas domiciliárias. Rev Saúde Publica. 1981; 15(5):543-50.

10. Carandina L, Sanches O, Carvalheiro JR. Análise das condições de saúde e de vida da população urbana de Botucatu, SP: I. Descrição do plano amostral e avaliação da amostra. Rev Saúde Publica. 1986; 20(6):465-74.

11. César CLG, Carandina L, Alves MCGP, Barros MBA, Goldbaum M. Saúde e condição de vida em São Paulo. São Paulo: USP; 2005.

12. Veras RP, Silva SD, Souza CAM, Milioli R, Ventura F. Proposta metodológica para inquérito domiciliar com populações idosas em um centro urbano do Estado do Rio de Janeiro (Brasil). Rev Saúde Pública. 1989; 23:429-38.

13. Valete-Rosalino CM, Rozenfeld S. Triagem auditiva em idosos: comparação entre auto-relato e audiometria. Rev Bras Otorrinolaringol. 2005; 71(2):193-200.

14. Newton VE, Macharia I, Mugwe P, Ototo B, Kan SW. Evaluation of the use of a questionnaire to detect hearing loss in Kenyan pre-school children. Int J Pediatr Otorhinolaryngol. 2001; 57(3):229-34.

15. Heathershaw KL, Wake M. Effectiveness of a parent questionnaire in the detection of hearing loss in school entry children. J Pediatr Child Health. 2000; 36(3):8-9.

16. Anderson MIP. Saúde e condições de vida do idoso no Brasil. Textos Envelhecimento. 1998; 1(1). Disponível em: URL: http://www.unati.uerj.br/tse/ 
scielo.php?script=sci_arttext\&pid=S1517-592819 $98000100002 \&$ lng=pt\&nrm=iso. Acesso em 18 set 2007.

17. Rosa TEC, Benicio MHD, Latorre MRDO, Ramos LR. Fatores determinantes da capacidade funcional entre idosos. Rev Saúde Pública. 2003; 37(1):40-8. 18. Cruickshanks KJ, Wiley TL, Tweed TS, Klein BEK, Klein R, Mares-Perlman JA et al. Prevalence of hearing loss in older adults in Beaver Dam, Wisconsin: the epidemiology of hearing loss study. Am J Epidemiol. 1998; 148(9):879-86.

19. Godinho RN, Gonçalves TML, Nunes FB, Becker CG, Becker HMG, Guimarães RES et al. Prevalence and impact of chronic otitis media in school age children in Brazil. First epidemiologic study concerning chronic otitis media in Latin America. Int J Pediatr Otorhinolaryngol. 2001; 61(3):223-32.

20. Piatto VB, Maniglia JV. Avaliação da audição em crianças de 3 a 6 anos em creches e pré-escolas municipais. J Pediatr. 2001; 77(2):124-30.

21. Béria JU, Raymann BC, Gigante LP, Figueiredo $A C$, Jotz G, Roithman R, et al. Hearing impairment and socioeconomic factors: a population-based survey of an urban locality in southern Brazil. Rev Panam Salud Publica. 2007; 21(6):381-7.

22. Sindhusake D, Mitchell P, Smith W, Golding M, Newall $P$, Hartley $D$, et al. Validation of self-reported hearing loss. The Blue Mountains hearing study. Int J Epidemiol. 2001, 30(6):1371-8.

23. Lepargneur $\mathrm{H}$. O novo quadro mundial da mulher. Mundo Saúde. 1999; 23(2):69-78.

24. Boltanski L. As classes sociais e o corpo. 3. ed. Rio de Janeiro: Graal; 1989.
25. Bazargan M, Bazargan-Hejazi S, Baker RS. Who is playing doctor? The gap between selfperceived versus professionally diagnosed chronic conditions among undeserved minority. Prev Med. 2005; 41(5-6):883-6.

26. National Council on the Aging. The consequences of untreated hearing loss in older persons. ORL Head Neck Nurs. 2000; 18(1):12-6.

27. Brasil. Ministério da Saúde. Promoção da saúde. Ministério da Saúde. Governo Federal: Brasília; 2001.

28. National Academy on an Aging Society. Hearing loss: a growing problem that affects quality of life. Challenges for the 21st Century: chronic and disabling conditions. 1999 (2). Disponível em: URL: http://ihcrp.georgetown.edu/agingsociety/pdfs/ hearing.pdf. Acesso em 06 jan, 2005.

29. Brasil. Ministério da Educação. Dados sobre número de deficientes auditivos no Brasil; 2005. Disponível em: URL: http://portal.mec.gov.br// seesp/index.php?option=content\&tash $=$ view\&id= 1638\&FlagNotícias $=1 \&$ ltemedid=1754. Acesso em 06 jun 2005.

30. Decaro JJ, Mudgett-Decaro PA, Dowaliby F. Attitudes toward occupations for deaf youth in Sweden. Am Ann Deaf. 2001; 146(1):51-9.

31. Guerra MR, Lourenço PMC, BustamanteTeixeira MT, Alves MJM. Prevalência perda auditiva induzida por ruído em empresa metalúrgica. Rev Saúde Publica. 2005; 39(2):238-44.

32. Alberti PW. The prevention of hearing loss worldwide. Scand Audiol Suppl. 1996; 42:15-9.

DOI: 10.1590 / S1516- 18462009005000009

RECEBIDO EM: 01/02/2008

ACEITO EM: 08/09/2008

Endereço para correspondência:

Mariana Sodário Cruz

Rua das Camélias, 139

Avaré - SP

CEP: $18706-410$

E-mail: msodario@yahoo.com.br 